\title{
Functional Brain Imaging of Young, Nondemented, and Demented Older Adults
}

\author{
Randy L. Buckner \\ Howard Hughes Medical Institute and Washington University \\ Abraham Z. Snyder, Amy L. Sanders, Marcus E. Raichle, \\ and John C. Morris
}

Washington University

\begin{abstract}
Brain imaging based on functional MRI (fMRI) provides a powerful tool for characterizing age-related changes in functional anatomy. However, between-population comparisons confront potential differences in measurement properties. The present experiment explores the feasibility of conducting fMRI studies in nondemented and demented older adults by measuring hemodynamic response properties in an event-related design. A paradigm involving repeated presentation of sensory-motor response trials was administered to 41 participants (14 young adults, 14 nondemented older adults, and 13 demented older adults). For half of the trials a single sensory-motor event was presented in isolation and in the other half in pairs. Hemodynamic response characteristics to the isolated events allowed basic response properties (e.g., amplitude and variance) between subject groups to be contrasted. The paired events further allowed the summation properties of the hemodynamic response to be characterized. Robust and qualitatively similar activation maps were produced
\end{abstract}

for all subject groups. Quantitative results showed that for certain regions, such as in the visual cortex, there were marked reductions in the amplitude of the hemodynamic response in older adults. In other regions, such as in the motor cortex, relatively intact response characteristics were observed. These results suggest caution should be exhibited in interpreting simple main effects in response amplitude between subject groups. However, across all regions examined, the summation of the hemodynamic response over trials was highly similar between groups. This latter finding suggests that, even if absolute measurement differences do exist between subject groups, relative activation change should be preserved. Designs that rely on group interactions between task conditions, parametric manipulations, or group interactions between regions should provide valuable data for making inferences about functional-anatomic changes between different populations.

\section{INTRODUCTION}

Brain imaging studies in young adults provide a foundation for exploring the functional anatomy of healthy aging and aging associated with progressive dementia. Completing such explorations may yield insights into the origins of age-associated cognitive change and perhaps even provide functional-anatomic markers that predict cognitive decline associated with Alzheimer's disease (e.g., Jonides, Marshuetz, Smith, Reuter-Lorenz, \& Koeppe, 2000; Reuter-Lorenz et al., 2000; Rypma \& D'Esposito, 2000; Backman et al., 1999; Backman, Almkvist, Nyberg, \& Andersson, 2000; Small, Perera, DeLaPaz, Mayeux, \& Stern, 1999; Smith et al., 1999; Madden et al., 1999; Cabeza et al., 1997; Mentis et al., 1996; Mentis et al., 1998; Schacter, Savage, Alpert, Rauch, \& Albert, 1996; Grady et al., 1993; Grady et al., 1994; Grady et al., 1995; Grady, McIntosh, Rajah, Beig, \& Craik, 1999). However, extending brain-imaging research into older adult popu-

lations brings with it several methodological challenges. The present study explores the feasibility of using functional MRI (fMRI) to study older adults.

Among the most serious challenges to fMRI feasibility is the possibility that the measures of neuronal activity used by fMRI have different properties in young and old adults. In particular, most fMRI studies are based on indirect measures of neural activity that rely on a coupling of local neuronal activity to a vascular hemodynamic response. Increased neural activity leads to changes in blood flow and volume without concordant changes in oxygen utilization. The result is a local increase in blood oxygen content that can be imaged with MRI (often called the blood oxygenation level dependent, or BOLD mechanism; Ogawa et al., 1992; Kwong et al., 1992). The BOLD mechanism thus depends on the integrity and proper regulation of local vasculature (Kuschinsky, 1999; Villringer, 1999). The first 
goal of the present study was to compare hemodynamic response properties as measured by BOLD-contrast fMRI in young, nondemented old, and demented old adults.

Several prior studies comparing hemodynamic response properties in young and older adults using fMRI have yielded mixed results (D'Esposito, Zarahn, Aguirre, \& Rypma, 1999; Taoka et al., 1998; Ross et al., 1997). Ross et al. compared sensory hemodynamic response properties in young and nondemented older adults in a blocked-task paradigm. Participants passively viewed a flickering red stimulus in 30-sec blocked epochs. Examining the percent signal change in activated voxels near striate and extrastriate visual cortex showed a significantly reduced response in old as compared to young adults (2.5\% vs. $4.0 \%)$. The response reduction was not likely due to fatigue or habituation, as the reduction was prominent within the first few seconds of the response epochs (see Figure 1 of Ross et al., 1997).

D'Esposito et al. investigated motor hemodynamic responses in young and old adults using an event-related task paradigm. In their study, participants visually fixated on a cross-hair that changed every $16 \mathrm{sec}$ to a small white circle. In response to the stimulus change, participants pressed buttons with both hands. Examining the percent signal change in activated voxels in or near motor cortex yielded similar response amplitudes between the old and young adults (see Figure 2 of D'Esposito et al., 1999). D'Esposito et al. did, however, note that the noise (variance) was significantly greater in the old as compared to young adults.

These two studies suggest opposing results. While both observed prominent neurally induced hemodynamic responses in older adults, Ross et al. (1997) observed a significant amplitude reduction in old relative to young adults, whereas D'Esposito et al. (1999) did not. One possibility for the opposing results is that the two studies examined different cortical regions, visual versus motor. A second goal of the present study was therefore to compare hemodynamic response properties in visual and motor cortex across young, nondemented old, and demented old adults.

A further issue needing exploration is the degree to which hemodynamic summation is comparable across populations. Hemodynamic response summation occurs when an initial hemodynamic response has not decayed prior to the initiation of a subsequent response. There are at least two reasons for examining hemodynamic response summation over sequential events in older adults. First, certain kinds of task paradigm, such as those based on rapid-presentation event-related fMRI (Clark, Maisog, \& Haxby, 1998; Dale \& Buckner, 1997), depend on roughly linear summation properties for their implementation. In older adults, the determination of whether such paradigms will be feasible requires exploring summation properties across overlapping events. Second, it is possible that in older adults the hemodynamic response is preserved (or preserved to a large degree) but nonetheless compromised when multiple repeated events are considered. Such a situation might arise if the hemodynamic reserve is limited in old adults compared to young adults. The difference between the results of D'Esposito et al. (1999) and Ross et al. (1997), for example, might be explained by the fact that the former considered the hemodynamic response to transient, isolated events, while the latter explored a summated response to continuous events. A final goal of the present study was to compare hemodynamic response summation properties across young, nondemented old, and demented old adults.

Several experimental design issues were considered in order to address these three goals. First, the Washington University Alzheimer's Disease Research Center clinically screened nondemented and demented older adult participants to meet appropriate inclusion criteria. Second, a simple sensory-motor paradigm was selected (adapted from Miezin, Maccotta, Ollinger, Petersen, \& Buckner, 2000). Participants viewed a transient large-field $8-\mathrm{Hz}$ flickering checkerboard and pressed a key at the onset of the flickering. In this manner, hemodynamic response characteristics could be examined in separate sensory (due to the visual stimulus) and motor (due to the keypress response) regions. Third, stimuli were either presented in isolation or in pairs. This manipulation allowed hemodynamic response properties to be characterized for isolated events, which are most relevant to event-related designs, as well as between-event summation properties, which are most relevant to blockedparadigm designs and rapid event-related paradigm designs (Dale \& Buckner, 1997). Finally, although not utilized fully in the present paper, multiple repetitions of high-resolution three-dimensional T1-weighted anatomic images were acquired on each subject to allow future analyses. Those analyses will compare anatomic differences between subject groups and relate functional response properties to anatomic differences.

\section{RESULTS}

\section{Behavior}

Behavioral data were acquired on 38 of 41 participants. For technical reasons, data were lost for two young adults and one demented older adult. Of 90 possible, the mean number of missed responses per participant were $0.33(<1 \%), 5.50(6.1 \%)$, and 3.25 (3.6\%) for the young, nondemented old, and demented old, respectively. Only four participants missed more than five responses (two nondemented and two demented older adults). Mean reaction times (computed from the individual subject medians) were $405(S E=16), 576(S E=$ $140)$, and $630 \mathrm{msec}(S E=147)$ for the young, nondemented old, and demented old, respectively. Although the reaction time data qualitatively showed a substantial slowing, the effect was not statistically significant $(F<1)$. 
The surprising lack of significance appears largely attributable to extreme variance and, in particular, two outlier values (one each from the demented and nondemented old adult groups). The effect of subject group approached significance when these two participants were removed $(F[2,33]=2.94, p=.07)$ and the difference between demented old and young subjects was significant in a post hoc $t$ test $(t[21]=2.46, p<.05)$. A nonparametric test including all subjects showed a significant difference between the demented old and young adults (Mann-Whitney test; $U=28, p<.05$ ).

\section{fMRI Activation Maps}

Activation maps for each subject group demonstrated prominent activation in visual and motor cortex (Figure 1). Activation included the left motor cortex (presumably due to motor response demands), striate cortex extending into extrastriate cortex (presumably due to visual processing demands), supplementary motor area, and right cerebellum. For many individual participants, including demented older adults, clear activations were detected in expected visual and motor regions. However, also noted was a clear reduction in the spatial extent and significance level of activations in the nondemented old as compared to young adults, and demented old as compared to nondemented old adults. To explore further the origins of these differences and gain a better understanding of hemodynamic response properties, regional signal change was examined in detail for visual and motor regions.

\section{Hemodynamic Response Amplitude}

To determine response amplitude in an unbiased manner, the mean selectively averaged responses from each subject group were plotted. The results are shown in Figure 2. Data are from a region in the visual cortex (seed coordinate $=3,-83,6$ located in or near the border of Brodmann's areas 17 and 18 in the Talairach and Tournoux (1988) atlas) and a region in the motor cortex (seed coordinate $-35,-31,58$ located in or near Brodmann's area 4).

For the visual cortex, clear reductions in response amplitude were present in the older adults relative to the younger adults ${ }^{1}$ (Figure 2). To quantify response amplitude and also to perform statistical tests, estimates of amplitude were obtained for each subject using the amplitude of the response at the peak time point (the third time point) minus baseline timepoints (the average of the first and last two time points). ${ }^{2}$ Results indicated a mean response of $1.20 \%, 0.59 \%$, and $0.62 \%$ for young, nondemented old, and demented old adults, respectively (Figure 3). ANOVA revealed a main effect of subject group on response amplitude for the visual region $(F[2,38]=7.28, p<.01)$. Post hoc $t$ tests
Figure 1. Statistical activation maps are displayed across transverse sections for each averaged subject group: (A) young adults, (B) nondemented older adults, and (C) demented older adults. Each set of images contains functional activation data from a single group superimposed on top of their averaged anatomic image in atlas space. Increasing color in the red-yellow direction indicates significantly increased response. Increasing color in the blue-green direction indicates significantly decreased response. The images use a statistical threshold of $p<.001$ $Z$ coordinate labels at the bottom indicate the approximate section based on the level in the Talairach and Tournoux (1988) atlas. Right is displayed on the right. Notably, all three groups show robust left motor cortex activation as well as visual cortex activation. However, activation in the young adults shows higher significance levels and a greater spatial extent above threshold.

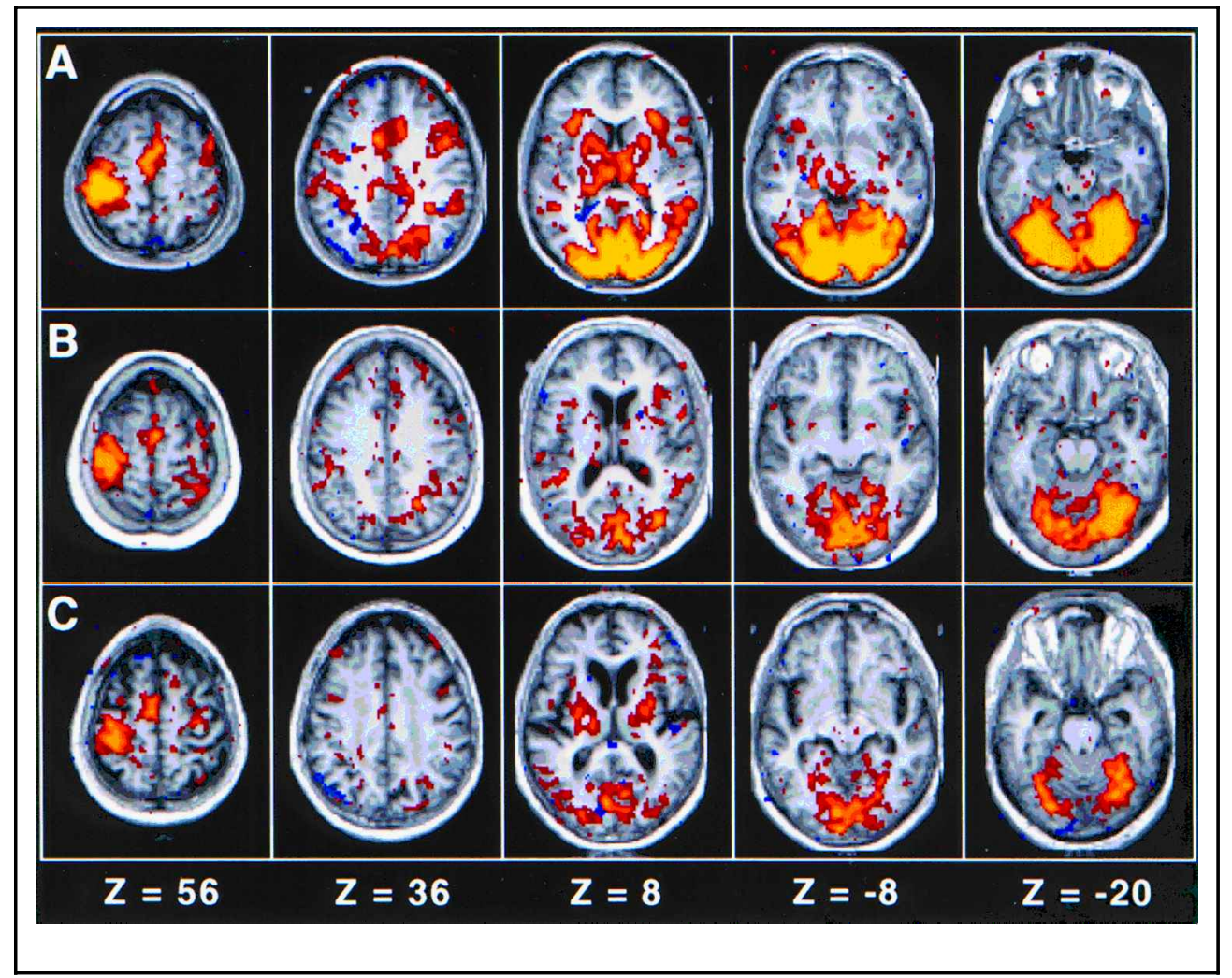




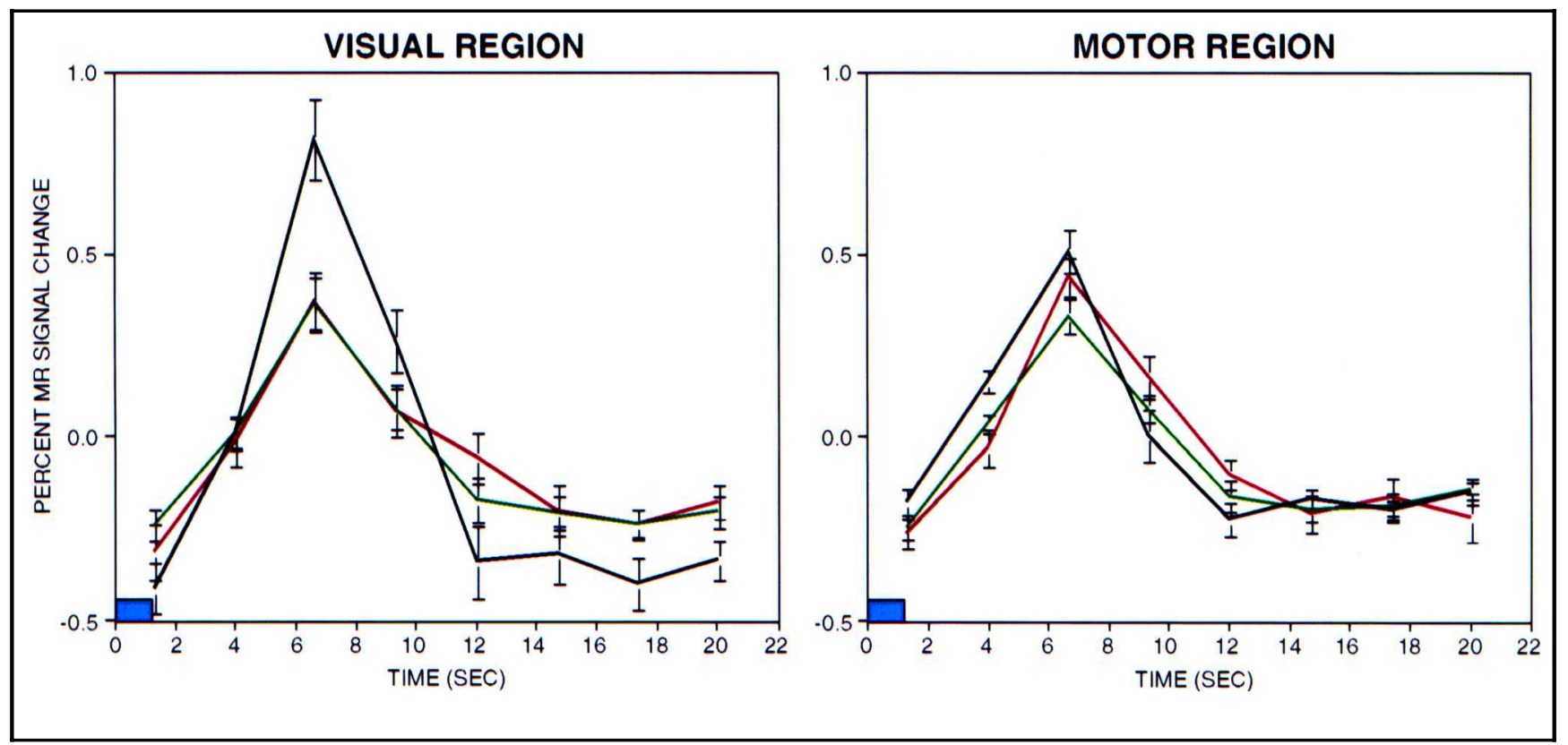

Figure 2. The selectively averaged hemodynamic responses are shown for visual (left panel) and motor (right panel) regions. Each panel displays mean data from each subject group with the line color indicating group: black $=$ young adults, green $=$ nondemented older adults, and red $=$ demented older adults. Error bars indicate standard error of the mean. The blue mark at the bottom of each panel represents when the visual stimulus was presented.

showed that nondemented old and demented old adults both were significantly reduced in amplitude as compared to young adults (unpaired $t[26]=3.17, p<.01$ and $t[25]=2.89, p<.01$, respectively).

For the motor cortex, similar response amplitudes were present across all groups. Applying the same methods as above, mean response amplitudes were $0.68 \%, 0.51 \%$, and $0.64 \%$ for young, nondemented old, and demented old adults, respectively (Figure 3). ANOVA did not reveal a main effect of subject group on response amplitude for the motor region $(F[2,38]=$ $1.44, p>.2)$. No differences reached significance in post hoc $t$ tests although the difference between young and nondemented old showed a trend $(t[26]=1.71, p=$ .10). A subtle shift in timing between groups was observed in the motor region (best observed in the return

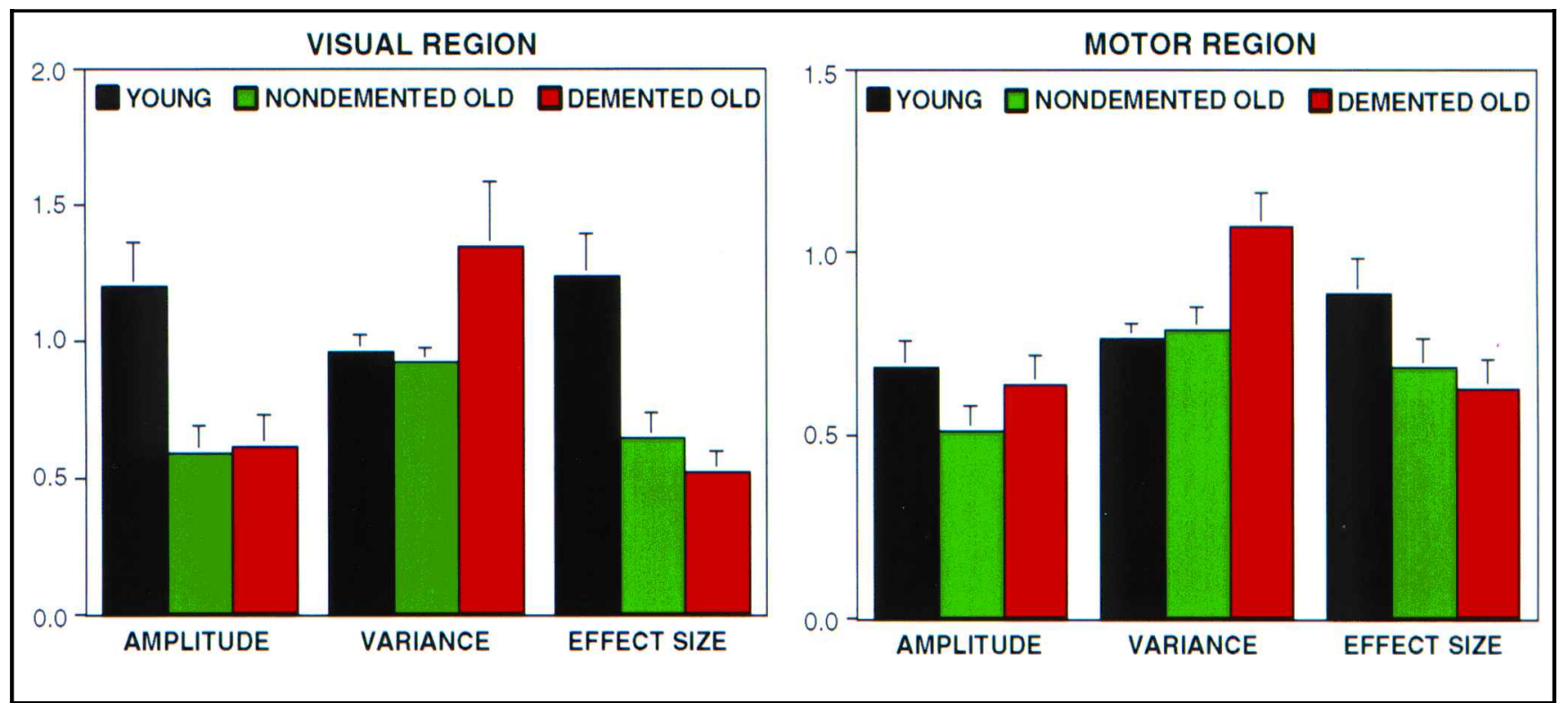

Figure 3. Descriptive statistics for the hemodynamic responses are plotted separately for visual (left panel) and motor (right panel) regions. Descriptive statistics include amplitude (in percent signal change), variance (in percent standard deviation), and effect size (unit-less). Each panel plots the mean descriptive data from each subject group with the bar color indicating group: black $=$ young adults, green $=$ nondemented older adults, and red $=$ demented older adults. Error bars indicate standard error of the mean. 
to baseline). Young adults were fastest, nondemented old next, and demented old adults slowest. This timing difference, which is noted here purely as an untested observation, may relate to the timing of the motor response.

The contrasting behavior of the visual and motor regions suggested the hemodynamic response amplitude varied between subject groups depending on which region was examined. This interaction effect was significant based on ANOVA entering region and group as two separate factors $(F[2,38]=4.00, p<.05)$. Thus, hemodynamic response amplitude in the visual region changed significantly more between young and old adults than did the response amplitude in the motor region.

\section{Hemodynamic Response Variance}

For the visual and motor regions described above, the within-subject standard deviation of the response was computed by averaging the separate standard deviations for each of the eight time points in the hemodynamic response epoch. ${ }^{3}$ The mean within-subject standard deviations for the visual region were $0.96 \%, 0.92 \%$, and $1.35 \%$ for the young, nondemented old, and demented old adults, respectively (Figure 3 ). ANOVA showed a trend for significance $(F[2,38]=2.75, p=.08)$. In posthoc $t$ tests, demented old adults showed a trend for more variance as compared to nondemented old adults (unpaired $t[25]=1.80, p=.08$ ). The mean standard deviations for the motor region were $0.76 \%, 0.79 \%$, and $1.07 \%$ for the young, nondemented old, and demented old adults, respectively (Figure 3 ). ANOVA showed a significant effect of subject group $(F[2,38]=5.92, p<$ $.01)$. Post hoc $t$ tests showed significantly more variance in demented old adults than nondemented old adults $(t[25]=2.51, p<.05)$ and young adults $(t[25]=3.03, p$ $<.01$ ). Thus, the within-subject variance (standard deviation) of the hemodynamic response was greatest in demented older adults.

To expand on these findings, the effect size was computed for each region by dividing the estimated amplitude of the hemodynamic response for each subject by his/her standard deviation. For the visual region, the mean effect sizes were $1.24,0.64$, and 0.52 for young, nondemented old, and demented old adults, respectively (Figure 3 ). The effect of subject group was significant by ANOVA $(F[2,38]=11.32, p<.001)$ and post hoc $t$ tests revealed that both older adult groups showed significantly lower effect sizes than the young adults $(t[26]=3.32, p<.01$ and $t[25]=4.07, p<.001$ for nondemented and demented old adults, respectively). The parallel analysis for the motor region revealed qualitatively similar results. Effect sizes were 0.89, 0.68, and 0.63 (Figure 3). This effect showed a trend towards significance $(F[2,38]=2.55, p=.09)$ with the post hoc $t$ test comparing young and demented old adults signifi- cant $(t[25]=2.09, p<.05)$. These changes in effect size may partly underlie the differences in the activation maps observed earlier (Figure 1).

\section{Comparison of Amplitude and Variance Between Regions}

Given the observed differences in amplitude and variance between subject groups, a natural set of questions to ask is whether such parameters covaried across regions which would suggest a global factor that varied over subjects. In other words, does a high hemodynamic response variance in one region predict a high variance in another? Does a high amplitude response in one region predict a high amplitude response in another? Amplitude did not correlate between visual and motor regions $\left(r^{2}=.03 ; F[1,38]=1.15, p=.29\right)$ while variance $\operatorname{did}\left(r^{2}=.34 ; F[1,38]=19.59, p<.001\right)$. These results suggest some subjects show more hemodynamic variance than others, and that this increase in variance is consistent across regions.

\section{Hemodynamic Response Summation}

To characterize summation properties, the hemodynamic responses from the one-trial conditions were subtracted from the two-trial conditions. The result was an estimate of the added contributions of the second, summated hemodynamic responses. These summated responses were then directly compared to the unsubtracted one-trial condition responses. To aid visualization, all responses were baseline shifted to a mean of zero. Such a shift is necessitated here because subtracted and unsubtracted time course data will obligatorily fall at different baseline values. Figure 4 displays the results. The added contributions of the second responses were highly similar to the isolated one-trial condition responses. Thus, the hemodynamic response summated in a similar fashion for all subject groups, independent of whether the absolute response amplitude was different (visual region) or similar (motor region) across groups.

To quantify more precisely the summation properties across groups, the amplitudes of the added hemodynamic response contributions were derived in an analogous manner to that used for the isolated amplitudes above. Results for the visual region were (numbers in parentheses are isolated trial amplitude estimates from above): $1.22 \%(1.20 \%), 0.59 \%$ (0.59\%), and $0.65 \%$ $(0.62 \%)$ for young, nondemented old, and demented old adults, respectively. Results for the motor region were: $0.65 \%(0.68 \%), 0.50 \%(0.51 \%)$, and $0.59 \%$ $(0.64 \%)$. Transforming these values into the percent amplitude of the added responses (relative to the isolated responses; $100 \%$ being perfect linear summation) yielded: $102 \%, 100 \%$, and $105 \%$ for young, nondemented old, and demented old adults, respectively, 

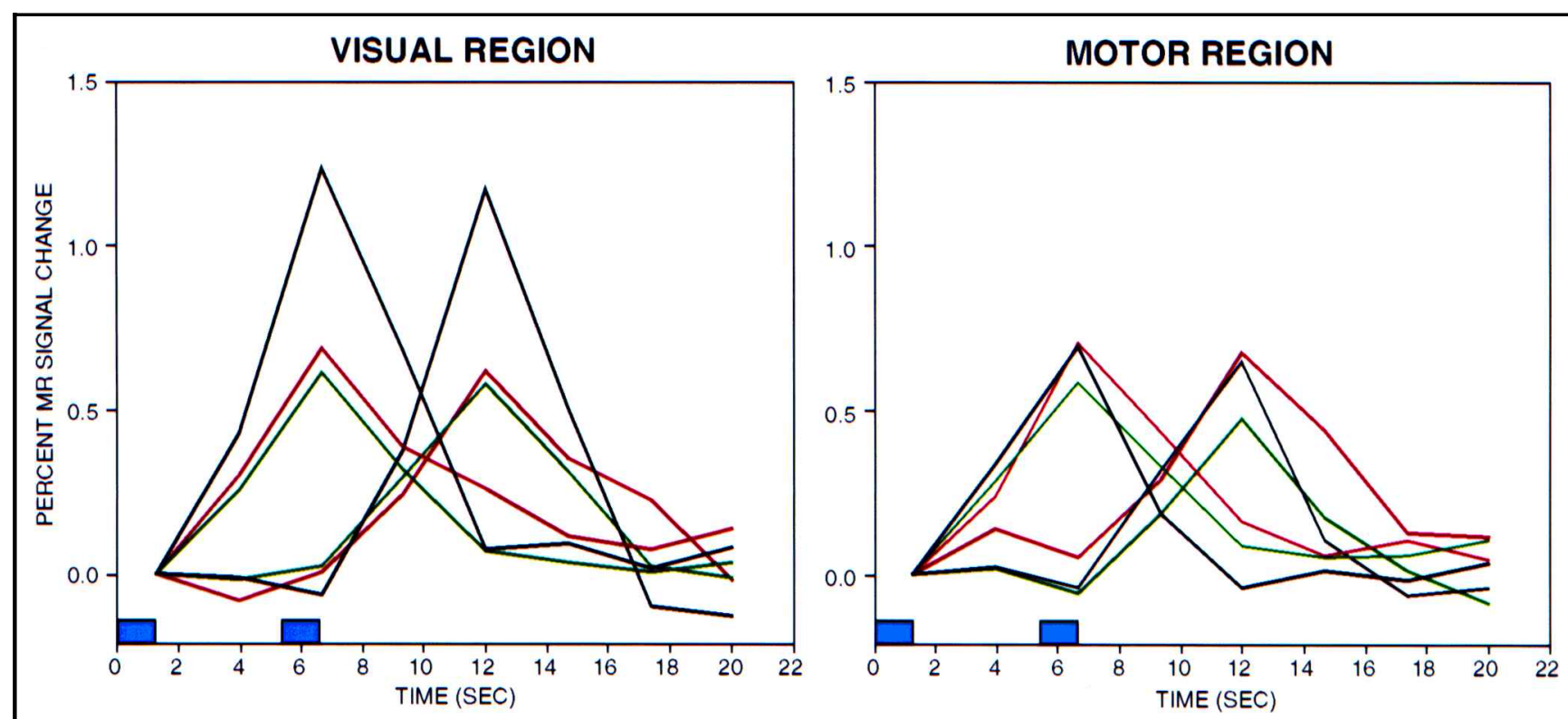

Figure 4. The selectively averaged hemodynamic responses are shown for visual (left panel) and motor (right panel) regions to illustrate the linear summation of the hemodynamic response. Each panel displays mean data from each subject group with line color indicating group: black = young adults, green $=$ nondemente $d$ older adults, and red $=$ demented older adults. The blue mark at the bottom of each panel represents when the two visual stimuli were present. The lines in each panel that show a peak to the left represent the selectively averaged data from the isolated trial events (similar to Figure 2). The lines that show a peak to the right represent the added contributions of the second (summated) trials in the two-trial condition. All responses are adjusted to start at a baseline of zero at their onset. Note the near linear summation across all groups for both regions, as indicated by similar amplitudes for the left and right peaks.

for the visual region and 96\%, $98 \%$, and $92 \%$ for the motor region.

\section{DISCUSSION}

Brain imaging based on fMRI provides a powerful method for examining functional-anatomy underlying ageassociated cognitive change. However, it will be necessary to understand potential differences in fMRI measurement properties between populations to fully exploit and interpret between-group comparisons. To begin such explorations, a simple sensory-motor fMRI paradigm was examined in three subject groups: young adults, nondemented older adults, and demented older adults. The main finding was quite optimistic: Imaging was successful and yielded robust activation of visual and motor regions in all subject groups (Figure 1). Thus, these data suggest that brain imaging based on fMRI is feasible in the study of older adults including demented individuals. Beyond this basic result, more detailed quantification of the data yielded several important considerations for imaging older adults. These findings relate to (1) the amplitude of the hemodynamic response, (2) the variance of the response, and (3) the summation properties of the response.

Significant reductions in the amplitude of the hemodynamic response were noted in older adults relative to younger adults, both for nondemented and demented subject groups. However, these reductions were region- ally specific: The visual region showed a significant amplitude reduction and the motor region did not. Moreover, the interaction between the two regions was significant. As noted in the Introduction, several prior studies have compared hemodynamic response amplitude between young and old adults. Of these, D'Esposito et al. (1999) and Ross et al. (1997) have yielded seemingly conflicting results. Ross et al. noted a significant amplitude reduction (attenuation) of the hemodynamic response in old adults, and D'Esposito et al. showed highly similar responses in old and young adults. The present results may help to clarify these earlier inconsistencies: Ross et al. (1997) examined hemodynamic response in the visual cortex while D'Esposito et al. (1999) examined the motor cortex.

What might regional variation in hemodynamic response properties reflect? One hypothesis is that there is regional variation in the coupling of neuronal activity to hemodynamic response. The present data are fully consistent with such a possibility. However, a second possibility is that there are regional differences in neural activity patterns. In our paradigm, visual cortex activity may be differentially affected in older adults because of either core sensory impairments or artifacts associated with imperfect stimulus viewing in older adults (e.g., differences in the ability to fixate). Mentis et al. (1996) and Mentis et al. (1998) provide data generally consistent with this interpretation. Mentis et al. observed that demented older adults, as compared to nondemented 
older adults, showed reduced visual cortex blood flow response to high frequency visual stimulation, suggesting a visual dysfunction in certain older adults. Our data, however, do not show a significant difference in visual cortex between nondemented and demented older adults as would be predicted by Mentis et al. (1998) . Given these uncertainties, the causes of the significant regional differences in hemodynamic response magnitude will have to await further study. The difference between regions may be related to regional differences in neural activity, coupling of vascular response to regional activity, or perhaps a combination of the two. Nonetheless, the present results suggest that hemodynamic response amplitude in certain regions can be preserved between young and old adults (as indicated by the motor region data) and that response amplitudes can be similar between demented and nondemented older adults.

Analysis of hemodynamic response variance showed demented older adults to have more variance than young or nondemented older adults. D'Esposito et al. (1999) have previously reported increased response variance in nondemented old as compared to young adults. Thus, both studies converge to suggest older adults can show significantly increased hemodynamic variance over young adults. The two studies differ in the degree to which a variance increase occurred in the nondemented older adults. One possible reason for this difference relates to participant recruitment. The nondemented older adults in the present study were extensively screened, highly motivated individuals who regularly participate in studies as part of the Alzheimer's Disease Research Center. These atypically healthy older adults, who showed variance in the general range of young adults, may represent the optimal imaging situation for older adults. Taken collectively, the two studies combine to suggest increased variance is an issue for imaging studies of older adults and particularly so for studies of demented participants.

Perhaps the most optimistic finding in the present study relates to the summation properties of the hemodynamic response across sequential events. All subject groups for both regions showed near linear summation of the response, independent of the level of the response (Figure 4). Such a finding suggests rapid-presentation event-related designs are possible in older adults (Dale \& Buckner, 1997). Moreover, as will be discussed below, the presence of intact summation properties across groups provides considerable inferential power to certain forms of between-group comparisons. One caveat to consider in interpreting these results is the limited range in which hemodynamic response summation was tested (events were spaced approximately $5 \frac{1}{2} \mathrm{sec}$ apart). Studies in young adults have shown nonlinearity in response properties at faster response rates (e.g., Huettel \& McCarthy, 2000; Friston, Josephs, Rees, \& Turner, 1997). Thus it will be important to explore further the boundary conditions for summation similarities between young and old adults in future studies.

\section{Implications for Experimental Design and Interpretation}

Imaging studies comparing young and old adults confront several challenges that the present results emphasize. As noted above, regional variation may exist in the coupling of the hemodynamic response, and differences in measured variance can be pronounced. For these reasons, we echo the concerns of D'Esposito et al. (1999) that main effects between subject groups may be difficult to interpret.

Direct between-group comparisons may lend themselves to interpretation errors because they assume that the absolute levels of hemodynamic response are the same between subject groups and because they also assume that the summation and/or scaling properties of the response are the same between groups. There are, nonetheless, some examples in the present data set and in D'Esposito et al. (1999) that meet or come close to these assumptions. Both D'Esposito et al. and the present study noted similar amplitude responses in old and young adults in the motor cortex. The present study extended this finding to suggest similar responses in nondemented and demented older adults. In fact, similar amplitude responses between demented and nondemented older adults were noted for both regions tested. Thus, under certain conditions, direct comparison of hemodynamic response levels between subject groups may be informative. However, the uncertainty concerning regional variation suggests that direct between-group comparisons in hemodynamic response amplitude should be interpreted extremely cautiously at best.

Given the uncertainty of whether the hemodynamic response amplitude is equivalent between young and old adults, a conservative approach is to adopt designs and analyses that do not rest on direct amplitude (magnitude) comparisons between subject groups. One analysis strategy that circumvents interpreting main effects is to interpret group-by-region interactions. In such analyses, interpretations will account for/consider the differences in one brain region's being more pronounced than those in another region. Overall main effects will not cause artifactual differences between groups (see D'Esposito et al., 1999 for a similar argument). However, such analysis assumes that, whatever the baseline difference in hemodynamic response, the hemodynamic response scales similarly across regions.

Another class of analyses that circumvent direct amplitude comparisons are those that depend on relative change across conditions within a group, rather than on absolute change between groups. Two related forms of 
such analyses are group-by-condition interactions and between-group parametric manipulations. Group-bycondition interactions are extremely promising because they rely on the relative change within one group's being significantly greater than the relative change in another group. Global effects of medication, health condition, atrophy, or other still-unconsidered factors would simply be handled by an overall offset with no contribution to the interaction effect. Moreover, the one consistent property noted in the present data, for all regions and subject groups, was that whatever the level of hemodynamic response, responses showed similar summation properties across subject groups. Thus, in employing a group-by-condition analysis, systematic differences between conditions in one group versus another would likely reflect true differences in underlying neural activity. For example, if the hemodynamic response doubles from one condition to the next in young adults but increases only minimally in old adults, the explanation is likely a genuine difference in brain activity between the two groups. Between-group parametric manipulations are a potentially powerful extension of this idea where more than two levels of a condition are considered in each group. Mentis et al. (1996) and Mentis et al. (1998) present excellent examples of between-group parametric designs employed in the study of sensory processing in older adults. The concept should extend to cognitive paradigms.

\section{METHODS}

\section{Subjects}

Forty-one right-handed, English-speaking individuals participated for $\$ 75$ payment. Fourteen young participants (five male) were recruited by advertisement from the student body of Washington University. The mean age of these young participants was 21.1 years (range: 18-24 years). A history of neurological or visual illness served as exclusion criteria. Twenty-seven older participants were recruited from the registry of the Washington University Alzheimer's Disease Research Center (ADRC). Fourteen (five male) were nondemented with a mean age of 74.9 years (range: 66-89 years). Thirteen (six male) were demented with a mean age of 77.2 years (range: 68-83 years). Older adults were excluded if they had neurologic, psychiatric, or medical illnesses that could cause dementia. All older adults had normal (corrected) visual acuity.

Dementia status was established using the ADRC's recruitment and assessment procedures (described in Berg et al., 1998). In brief, nondemented control subjects and those with mild dementia of the Alzheimer's type (DAT) were assessed clinically with the Clinical Dementia Rating (CDR), where CDR 0 indicates no dementia and CDR 0.5 and CDR 1 indicate very mild and mild DAT, respectively (Morris, 1993). The
ADRC diagnostic criteria for DAT are comparable to those for "probable $\mathrm{AD}$ " as described by the Work Group of the National Institute of Neurological and Communicative Disorders and Stroke and Alzheimer's Disease and Related Disorders Association (McKhann et al., 1984). The diagnostic accuracy for DAT as confirmed by the neuropathologic presence of $\mathrm{AD}$ is $93 \%$, including for subjects in the earliest stages of dementia (i.e., CDR 0.5) (Berg et al., 1998). Of the 13 demented participants, 8 had very mild DAT (CDR 0.5) and 5 had mild DAT (CDR 1). All nondemented older adults had CDR 0.

\section{MRI Acquisition Procedures}

Scans were conducted on a Siemens 1.5-T Vision System (Erlangen, Germany) with a standard circularly polarized head coil. A pillow and thermoplastic facemask were used to minimize head movement. Participants' heads were positioned low in the head coil (toward their feet) so that the cerebral cortex fell within the center of the head coil (i.e., participants were typically landmarked about 1 in. above their eyebrows). Headphones dampened scanner noise and allowed communication with subjects.

In order to provide high-resolution structural images with high gray-white contrast, a series of three to four separate T1-weighted MP-RAGE anatomic images were acquired per subject (resolution $=1 \times 1 \times 1.25 \mathrm{~mm}$, TR $=9.7 \mathrm{msec}, \mathrm{TE}=4 \mathrm{msec}$, flip angle $=10^{\circ}, \mathrm{TI}=20 \mathrm{msec}$, $\mathrm{TD}=500 \mathrm{msec}$ ). The first structural image of the series was acquired at the beginning of the session followed by two to three additional structural image acquisitions at the end of the session, following the functional data acquisition. The structural data were motion-corrected to one another using rigid-body realignment (Snyder, 1996) and interpolated to isotropic 1-mm voxels (for detailed anatomic examination) and $2-\mathrm{mm}$ voxels (for use as a backdrop for functional imaging). Although not fully utilized in the present manuscript, the anatomic data are sufficient for anatomic quantification, including surface reconstruction and white/gray/CSF measurements (e.g., Snyder, Sanders, Linenweber, Morris, \& Buckner, 2000).

Functional images were collected with an asymmetric spin-echo sequence sensitive to BOLD-contrast (volume $\mathrm{TR}=2.68 \mathrm{sec}, 3.75 \times 3.75 \mathrm{~mm}$ in-plane resolution; $\mathrm{T}^{*} *$ evolution time $=50 \mathrm{msec}$, alpha $=90^{\circ}$; Conturo et al., 1996). Whole-brain imaging was performed using 16 contiguous 8 -mm thick slices acquired parallel to the plane of the anterior-posterior commissure. For each subject, four separate functional image runs were acquired, with each run accumulating 128 total sequential whole-brain image acquisitions. Each run lasted approximately $5.5 \mathrm{~min}$ and a 2 -min delay existed between runs, during which subjects were permitted to rest. 


\section{Behavioral Procedures}

Stimulus display was controlled by a Power Macintosh computer (Apple, Cupernico, CA) using PsyScope software (Cohen, MacWhinney, Flatt, \& Provost, 1993). Keypress responses were recorded using a fiber-optic light-sensitive keypress connected to a PsyScope button box (Carnegie Mellon University, Pittsburgh, PA). All buttons except one were physically covered to minimize response complexity. Stimuli were rear projected (AmPro Model LCD-150, Ampro, Melbourne, FL) onto a screen placed at the back of the magnet bore. Participants viewed the screen through a mirror fastened to the top of the head coil. Participants requiring corrective lenses (mostly older adults) were supplied magnetcompatible glasses.

The basic task paradigm consisted of presentation of a 1.5-sec duration visual stimulus. Participants pressed a key with their right index fingers upon stimulus onset. The visual stimulus was an 8-Hz counterphase flickering (black to white) checkerboard subtending approximately $12^{\circ}$ of visual angle ( $6^{\circ}$ in each visual field). Stimulus parameters were identical to those used by Miezin et al. (2000). The stimulus onset was triggered at the beginning of the image acquisition via the PsyScope button box. Spatial frequency of the checkerboard decreased with visual angle to be approximately constant in relation to acuity across the visual field.

Runs were structured such that for every eight-image acquisition $(21.44 \mathrm{sec})$, one of two kinds of trial condition were presented ( 15 trials per run for a total of 60 trials per subject). Task trials either involved stimuli presented in isolation (one-trial condition) or in pairs with an inter-trial interval of $5.36 \mathrm{sec}$ (two-trial condition). One-trial and two-trial conditions were pseudorandomly intermixed such that eight trials of one type and seven of the other appeared in each run. The logic of this design (Dale \& Buckner, 1997) is that the onetrial conditions can be examined to determine the evoked hemodynamic response to an isolated, transient event. The two-trial conditions further allowed the summation properties of the hemodynamic response to be examined: To the degree that the added responses in the two-trial conditions were similar to the responses in the one-trial conditions, the hemodynamic dynamic response exhibits linear summation. Four image acquisitions involving only fixation were acquired prior to the first trial and following the last trial in each run.

\section{fMRI Data Analysis}

For each fMRI run, data were first preprocessed to remove several sources of noise and artifact using previously applied procedures (Wheeler, Petersen, \& Buckner, 2000; Konishi, Wheeler, Donaldson, \& Buckner, 2000). For all analyses, the first four image volumes were discarded to allow longitudinal magnetization sta- bilization. All functional image runs were corrected for odd/even slice-intensity differences and normalized to a whole-brain global signal intensity of 1000 using a single scaling factor per run. Motion correction using a rigidbody rotation and translation correction was then applied within each functional run (across sequential volume image acquisitions) and between functional runs within each subject (Snyder, 1996). All image volumes were interpolated and spatially scaled to conform to the Talairach and Tournoux (1988) atlas (interpolated to 2$\mathrm{mm}$ isotropic voxels). The atlas transformation was verified for each individual subject by plotting his/her spatially scaled anatomy image against the averaged template. Finally, the slope and mean were subtracted from each voxel to remove effects of linear drift and also subtract signal variation due to underlying structural anatomy. The mean voxel intensity was saved and later used to scale effects to percent signal change.

The functional (BOLD) responses were analyzed in two phases. First, individual subject and group analyses were performed in an event-related fashion using selective-averaging methods (Buckner et al., 1998; Dale \& Buckner, 1997). For this analysis, data were spatially smoothed using a one-voxel Hanning filter and then sorted based on the onset of trial events. The one-trial and two-trial event conditions were separately averaged. For each subject, all one-trial conditions were averaged together to produce a mean hemodynamic response at each voxel for the isolated trials events. The mean response was calculated over eight image acquisitions (21.44 sec given the TR of $2.68 \mathrm{sec}$ ). All two-trial conditions were similarly averaged. For each condition within each subject, the variance (standard deviation) across events was also computed. To determine the added contribution of the second event in the two-trial conditions, the one-trial conditions were simply subtracted from the two-trial conditions. Statistical activation maps based on a $t$ statistic were constructed for the selectively averaged data by regressing the data at each voxel against a family of possible hemodynamic responses based on a gamma function (Buckner et al., 1998; Dale \& Buckner, 1997; Boynton, Engel, Glover, \& Heeger, 1996). For group-averaged images, the selectively averaged data were themselves averaged over subjects prior to statistical map generation.

Second, evoked hemodynamic responses were extracted for each subject from a priori defined regions and directly compared using a random-effects statistical model. The regions were derived by combining data across all subject groups and constructing a single composite activation map. Peak activations in visual and motor cortex were then derived from this composite activation map. Regions were defined to include all significant voxels within $12 \mathrm{~mm}$ of the locations of the peak activation seed points. In this manner, all subject groups contributed equally to region definition in order not to bias region definition to any particular subject 
group under the null hypothesis that the groups are the same. Nonetheless, bias could still be present if differences existed across groups. One subject group (via their level of activation) could bias the peak location selected and augment observed differences. ${ }^{4}$ The hemodynamic response and variance were obtained for each region for each subject and entered into subsequent analyses based on ANOVA and post hoc two-tailed $t$ tests.

\section{Acknowledgments}

We thank the staff of the Washington University ADRC for recruiting participants, Bill Kelley for help in implementing behavioral procedures, and Bill Powers for discussion. David Donaldson, Bonnie Carr, and two reviewers provided thoughtful comments on an early version of this manuscript. Tom Conturo and Erbil Akbudak provided assistance in the development of imaging procedures. This work was supported by National Institute of Aging grants AG05681, AG03991, National Institute of Mental Health grant MH57506, and a James S. McDonnell Foundation Program in Cognitive Neuroscience grant (99-63/9900003).

Reprint requests should be sent to Randy L. Buckner, Department of Psychology, Washington University, Campus Box 1125, One Brookings Drive, St. Louis, MO 63130, USA.

The data reported in this experiment have been deposited in National fMRI Data Center (http://www.fmridc.org). The accession number is 2-2000-1118w.

\section{Notes}

1. The apparent shift in baseline in the visual region between groups is a byproduct of baseline definition that, in this instance, is simply the run mean. When defined in this manner, larger responses extend symmetrically above and below the zero point to a greater degree than smaller responses. Alternative procedures, such as estimating a response baseline, assume a response profile and do not change the shape of the response. For these reasons, the raw averaged responses are displayed.

2. Model fitting procedures to estimate amplitude, such as those used by Miezin et al., 2000 and Boynton et al., 1996 proved too unstable for use here, likely because of the reduced signal-to-noise in some subjects and the relatively sparse temporal sampling.

3. Surprisingly, the standard deviation did not vary across the eight-image estimate of the hemodynamic response. For this reason, the mean estimate was used. The finding of constant variance over the evolution of the hemodynamic response constrains possible origins of the noise. The variance is not likely to originate from different amplitude responses that vary from trial to trial. Rather, the variance more likely originates from noise properties that are relatively constant across the fMRI run but nonetheless interfere with hemodynamic response estimation.

4. An alternative approach to region definition was piloted. In this pilot analysis, regions were defined on each individual subject based on his or her activation map. The results were qualitatively similar to those obtained in the present analysis. However, such an approach was abandoned because it may bias the absolute magnitude estimates. If individuals differ in variance, those participants with greater variance will bias selection of voxels with greater mean responses (these voxels will be more likely to reach significance). The approach we used, in which regions were defined on the combined activation map, was less sensitive to this form of bias. The statistical power was such that almost all voxels within the chosen radius of the peak were included. Nonetheless, if significant variability in activation location exists between subject groups, the seed location for the region may be biased to one group over another. In the data presented here, this was not likely the case (see Figure 1). However, the approach cannot be generalized to all situations without consideration of potential bias in region location.

\section{REFERENCES}

Backman, L., Almkvist, O., Nyberg, L., \& Andersson, J. (2000). Functional changes in brain activity during priming in Alzheimer's disease. Journal of Cognitive Neuroscience, 12, 134-141.

Backman, L., Andersson, J. L., Nyberg, L., Winblad, B., Nordberg, A., \& Almkvist, O. (1999). Brain regions associated with episodic retrieval in normal aging and Alzheimer's disease. Neurology, 52, 1861-1870.

Berg, L., McKeel, D. W., Miller, J. P., Storandt, M., Rubin, E. H., Morris, J. C., Baty, J., Coats, M., Norton, J., Goate, A. M., Price, J. L., Gearing, M., Mirra, S. S., \& Saunders, A. M. (1998). Clinicopathological studies in cognitively healthy aging and Alzheimer's disease: Relation of histologic markers to dementia severity, age, sex, and APOE genotype. Archives of Neurology, 55, 326-335.

Boynton, G. M., Engel, S. A., Glover, G. H., \& Heeger, D. J. (1996). Linear systems analysis of functional magnetic resonance imaging in human V1. Journal of Neuroscience, 16, 4207-4221.

Buckner, R. L., Goodman, J., Burock, M., Rotte, M., Koutstaal, M., Schacter, D. L., Rosen, B., \& Dale, A. M. (1998). Functional-anatomic correlates of object priming in humans revealed by rapid presentation event-related fMRI. Neuron, 20, 285-296.

Cabeza, R., Grady, C. L., Nyberg, L., McIntosh, A. R., Tulving, E., Kapur, S., Jennings, J. M., Houle, S., \& Craik, F. I. M. (1997). Age-related differences in neural activity during memory encoding and retrieval: A positron emission tomography study. The Journal of Neuroscience, 17, 391-400.

Clark, V. P., Maisog, J. M., \& Haxby, J. V. (1998). fMRI study of face perception and memory using random stimulus sequences. Journal of Neurophysiology, 79, 3257-3265.

Cohen, J. D., MacWhinney, B., Flatt, M., \& Provost, J. (1993). PsyScope: A new graphic interactive environment for designing psychology experiments. Behavioral Research Methods, Instruments, and Computers, 25, 257-271.

Conturo, T. E., McKinstry, R. C., Akbudak, E., Snyder, A. Z., Yang, T. Z., \& Raichle, M. E. (1996). Sensitivity optimization and experimental design in functional magnetic resonance imaging. Society for Neuroscience Abstracts, 22, 7.

Dale, A. M., \& Buckner, R. L. (1997). Selective averaging of rapidly presented individual trials using fMRI. Human Brain Mapping, 5, 329-340.

D'Esposito, M., Zarahn, E., Aguirre, G. K., \& Rypma, B. (1999). The effect of normal aging on the coupling of neural activity to the BOLD hemodynamic response. Neuroimage, 10, 6-14.

Friston, K. J., Josephs, O., Rees, G., \& Turner, R. (1997). Nonlinear event-related responses in fMRI. Magnetic Resonance in Medicine, 39, 41-52.

Grady, C. L., Haxby, J., Horwitz, B., Gillette, J., Salerno, J., Gonzalez-Aviles, A., Carson, R., Herscovitch, P., Schapiro, M., \& Rapoport, S. (1993). Activation of cerebral blood flow during a visuoperceptual task in patients with Alzheimertype dementia. Neurobiology of Aging, 14, 35-44. 
Grady, C. L., Maisog, J. M., Horwitz, B., Ungerleider, L. G., Mentis, M. J., Salerno, J. A., Pietrini, P., Wagner, E., \& Haxby, J. V. (1994). Age-related changes in cortical blood flow activation during visual processing of faces and locations. Journal of Neuroscience, 14, 1450-1462.

Grady, C. L., McIntosh, A., Horwitz, B., Maisog, J. M., Ungerleider, L. G., Mentis, M. J., Pietrini, P., Schapiro, M. B., \& Haxby, J. V. (1995). Age-related reductions in human recognition memory due to impaired encoding. Science, 269, 218-221.

Grady, C. L., McIntosh, A. R., Rajah, M., Beig, S., \& Craik, F. (1999). The effects of age on the neural correlates of episodic encoding. Cerebral Cortex, 9, 805-814.

Huettel, S., \& McCarthy, G. (2000). Evidence for a refractory period in the hemodynamic response to visual stimuli as measured by MRI. Neuroimage, 11, 547-553.

Jonides, J., Marshuetz, C., Smith, E. E., Reuter-Lorenz, P. A., \& Koeppe, R. A. (2000). Age differences in behavior and PET activation reveal differences in interference resolution in verbal working memory. Journal of Cognitive Neuroscience, 12, 188-196.

Konishi, S., Wheeler, M. E., Donaldson, D. I., \& Buckner, R. L. (2000). Neural correlates of episodic retrieval success. Neuroimage, 12, 276-286.

Kuschinsky, W. (1999). Regulation of cerebral blood flow. In C. Moonen \& P. Bandettini (Eds.), Functional MRI (pp. 15-23). New York: Springer.

Kwong, K. K., Belliveau, J. W., Chesler, D. A., Goldberg, I. E., Weisskoff, R. M., Poncelet, B. P., Kennedy, D. N., Hoppel, B. E., Cohen, M. S., \& Turner, R. (1992). Dynamic magnetic resonance imaging of human brain activity during primary sensory stimulation. Proceedings of the National Academy of Sciences, U.S.A., 89, 5675-5679.

Madden, D., Gottlob, L., Denny, L., Turkington, T., Provenzale, J., Hawk, T., \& Coleman, R. (1999). Aging and recognition memory: Changes in regional cerebral blood flow associated with components of reaction time distributions. Journal of Cognitive Neuroscience, 11, 511-520.

McKhann, G., Drachman, D., Folstein, M., Katzman, R., Price, D., \& Stadlan, M. (1984). Clinical diagnosis of Alzheimer's disease: Report of the NINCDS-ADRDA Work Group under the auspices of the Department of Health and Human Services Task Force on Alzheimer's Disease. Neurology, 34, 39-44.

Mentis, M., Alexander, G., Krasuski, J., Pietrini, P., Furey, M., Schapiro, M., \& Rapoport, S. (1998). Increasing required neural response to expose abnormal brain function in mild versus moderate or severe Alzheimer's disease: PET study using parametric visual stimulation. American Journal of Psychiatry, 155, 785-794.

Mentis, M., Horwitz, B., Grady, C. L., Alexander, G., VanMeter, J., Maisog, J., Pietrini, P., Schapiro, M., \& Rapoport, S. (1996). Visual cortical dysfunction in Alzheimer's disease evaluated with a temporally graded "stress test" during PET. American Journal of Psychiatry, 153, 32-40.
Miezin, F., Maccotta, L., Ollinger, J., Petersen, S., \& Buckner, R.L. (2000). Characterizing the hemodynamic response: Effects of presentation rate, sampling procedure, and the possibility of ordering brain activity based on relative timing. Neuroimage, 11, 735-759.

Morris, J. C. (1993). Clinical dementia rating. Neurology, 43, 2412-2414.

Ogawa, S., Tank, D. W., Menon, R., Ellerman, J. M., Kim, S. G., Merkle, H., \& Ugurbil, K. (1992). Intrinsic signal changes accompanying sensory stimulation: Functional brain mapping with magnetic resonance imaging. Proceedings of the National Academy of Sciences, U.S.A., 89, 5951-5955.

Ross, M. H., Yurgelun-Todd, D. A., Renshaw, P. F., Maas, L. C., Mendelson, J. H., Mello, N. K., Cohen, M. D., \& Levin, J. M. (1997). Age-related reduction in functional MRI response to photic stimulation. Neurology, 48, 173-176.

Rypma, B., \& D'Esposito, M. (2000). Isolating the neural mechanisms of age-related changes in human working memory. Nature Neuroscience, 3, 509-515.

Schacter, D. L., Savage, C. R., Alpert, N. M., Rauch, S. L., \& Albert, M. S. (1996). The role of hippocampus and frontal cortex in age-related memory changes: A PET study. Neuroreport, 7, 1165-1169.

Small, S. A., Perera, G. M., DeLaPaz, R., Mayeux, R., \& Stern, Y. (1999). Differential regional dysfunction of the hippocampal formation among elderly with memory decline and Alzheimer's disease. Annals of Neurology, 45, 466-472.

Smith, C., Andersen, A., Kryscio, R., Schmitt, F., Kindy, M., Blonder, L., \& Avison, M. (1999). Altered brain activation in cognitively intact individuals at high risk for Alzheimer's disease. Neurology, 53, 1391-1396.

Snyder, A. Z. (1996). Difference image versus ratio image error function forms in PET-PET realignment. In D. Bailey \& T. Jones (Eds.), Quantification of brain function using PET. San Diego: Academic Press.

Snyder, A. Z., Sanders, A. L., Linenweber, W., Morris, J. C., \& Buckner, R. L. (2000). Automatic atrophy assessment (AAA) in young, middle-aged, nondemented and demented older adults. Journal of Cognitive Neuroscience, Suppl. 2.

Talairach, J., \& Tournoux, P. (1988). Co-planar stereotaxic atlas of the buman brain. New York: Thieme.

Taoka, T., Iwasaki, S., Uchida, H., Fukusumi, A., Nakagawa, H., Kichikawa, K., Takayama, K., Yoshioka, T., Takewa, M., \& Ohishi, H. (1998). Age correlation of the time lag in signal change on EPI-fMRI. Journal of Computer Assisted Tomography, 22, 514-517.

Villringer, A. (1999). Physiological changes during brain activation. In C. Moonen \& P. Bandettini (Eds.), Functional MRI (pp. 3-13). New York: Springer.

Wheeler, M. E., Petersen, S. E., \& Buckner, R. L. (2000). Memory's echo: Vivid remembering reactivates sensory-specific cortex. Proceedings of the National Academy of Sciences, U.S.A., 97, 11125-11129. 